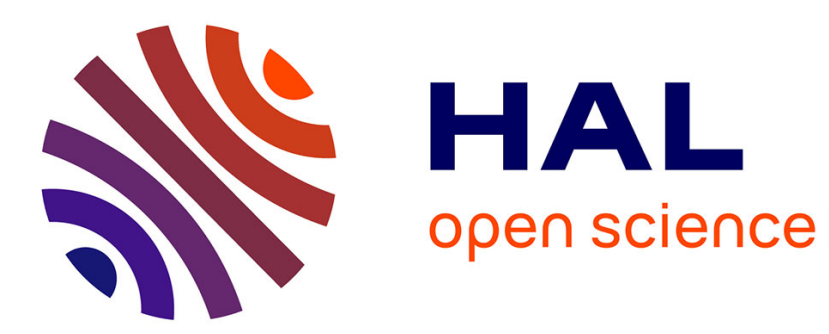

\title{
Dialogisme, parcours et altérité notionnelle : pour une intégration en langue du dialogisme ? \\ Sylvie Mellet
}

\section{To cite this version:}

Sylvie Mellet. Dialogisme, parcours et altérité notionnelle : pour une intégration en langue du dialogisme ?. Langue française, 2009, Dialogisme et marqueurs grammaticaux, 163, pp.157-173. 10.3917/lf.163.0157 . hal-01250513

\section{HAL Id: hal-01250513 \\ https://hal.science/hal-01250513}

Submitted on 4 Jan 2016

HAL is a multi-disciplinary open access archive for the deposit and dissemination of scientific research documents, whether they are published or not. The documents may come from teaching and research institutions in France or abroad, or from public or private research centers.
L'archive ouverte pluridisciplinaire HAL, est destinée au dépôt et à la diffusion de documents scientifiques de niveau recherche, publiés ou non, émanant des établissements d'enseignement et de recherche français ou étrangers, des laboratoires publics ou privés. 


\section{Dialogisme, parcours et altérité notionnelle : pour une intégration en langue du dialogisme?}

Sylvie MELLET (BCL, Université de Nice-Sophia Antipolis, CNRS, MSH de Nice)

\section{Enjeux et objectif}

Le dialogisme a-t-il une place en langue ? Ou, plus exactement, le dialogisme qui - si l'on en croit Bakhtine - ne relève pas de la linguistique, mais de ce qu'il appelle la «translinguistique ${ }^{1}$, est-il un fait purement discursif sans aucun soubassement linguistique, ou a-t-il un support en langue ? L'hypothèse qui sous-tend cette contribution est que le dialogisme, cette présence dans un énoncé d'autres voix porteuses d'énoncés auxquels l'énoncé en cours fait écho, est à ce point inhérent au langage humain qu'il ne saurait reposer sur de simples effets contextuels liés à l'actualisation en discours, mais qu'il doit nécessairement s'appuyer, en amont de la production langagière, sur des opérations énonciatives stables qui ont intégré et formalisé en langue ce processus dialogique. Mon objectif sera donc de cerner ces opérations et d'analyser leurs rapports avec le fait dialogique. Car même si le dialogisme est un phénomène très couvrant et polymorphe, son expression et son interprétation sont nécessairement portées par des marqueurs, dont certains manifestent avec lui des affinités privilégiées ; dans le cadre de la Théorie des Opérations Enonciatives (désormais TOE) développée par A. Culioli et à laquelle je me réfère, les marqueurs sont conçus comme les traces d'opérations énonciatives primaires, qui ont un statut linguistique et dont les diverses configurations définissent le signifié de base de chaque marqueur. On voit ici en quoi le questionnement liminaire va pouvoir alimenter une réflexion sur les liens entre dialogisme et grammaire : car, sans occulter que le dialogisme traverse aussi le lexique ${ }^{2}$, on constate que la liste des marqueurs privilégiés jusqu'ici répertoriés est principalement constituée de

\footnotetext{
${ }^{1}$ « Les rapports dialogiques [...] sont un objet de la translinguistique. [...] Dans la langue, objet de la linguistique, n'existe et ne peut exister aucun rapport dialogique » La poétique de Dostoïevski [1963] (trad. I. Kolitcheff), Paris, Seuil, 1970, p. 239. Cité par Vladimir Mikhaïlovitch Alpatov in R. Therkelsen, N. Møller Andersen et H. Nølke (éds.), 2007 : 209.
}

${ }^{2}$ Cf. Siblot (2001): 《De la dénomination à la nomination. Les dynamiques de la signifiance nominale et le propre du nom 》, Cahiers de praxématique $36: 189-214$. 
marqueurs grammaticaux ${ }^{3}$. La réponse à la question initialement posée passera donc par l'étude de quelques-uns d'entre eux et par la recherche de ce que Culioli appelle leur "forme schématique », dans l'espoir de mettre au jour ce dont ils sont constamment et unanimement la trace : leur éventuel point commun pourra, s'il est sémantiquement et énonciativement pertinent, fournir l'ancrage linguistique du dialogisme. Conséquemment, cet examen permettra peut-être de mieux appréhender où passe la frontière entre ce qui, au sein de la grammaire est nécessairement dialogique et ce qui peut ne pas l'être.

\section{Cadre théorique et choix terminologiques}

La notion bakhtinienne de dialogisme a été présentée en introduction de ce numéro, je n'y reviens donc pas. En revanche, il convient de préciser que, sans être aussi stricte que Bres et Nowakowska, pour qui l'énoncé [e] «rapporté » [i.e. dont l'énoncé dialogique se fait l'écho] a nécessairement, pour le locuteur, « le statut d'un énoncé actualisé ${ }^{4}$ » (2006: 29), je me situe néanmoins dans cette même mouvance qui confère au dialogisme la vertu de donner à entendre, non pas seulement des points de vue, mais des énoncés assertables, c'est-à-dire susceptibles d'être pris en charge par un énonciateur. Je reviendrai ultérieurement sur cette notion de prise en charge, cruciale pour mon propos. Convenons seulement, pour l'instant, que le dialogisme au sens strict, par lequel un énoncé répond à d'autres " énoncés qui l'ont précédé ou qui lui succèderont » (Bakhine 1952/1979/1984b : 355) ne saurait se contenter de prendre appui sur des contenus sans expression, sur de simples signifiés propositionnels désincarnés.

L'autre postulat théorique a déjà été évoqué en introduction : toute opération énonciative laisse une trace en discours sous la forme de divers marqueurs, lexicaux et grammaticaux, qui fournissent au linguiste les observables à partir desquels il peut reconstruire un modèle méta-linguistique susceptible de rendre compte de l'opération initiale, en elle-même inatteignable et inobservable par le linguiste. C'est donc en partant de l'étude d'un certain nombre de marqueurs grammaticaux réputés pour être associés de façon particulièrement fréquente et transparente à l'expression du dialogisme que l'on peut espérer modéliser les quelques opérations cognitivo-linguistiques qui sont à la base de celui-ci et sont susceptibles de rendre compte - parmi d'autres modèles possibles - de la puissance dialogique de ces marqueurs. On commencera par deux marqueurs

\footnotetext{
${ }^{3}$ Voir par exemple Bres 1999 et 2007.

${ }^{4}$ C'est ce terme «actualisé » qui me paraît ici excessif - sauf à entendre simplement par là que la relation prédicative « rapportée » est bien repérée par rapport à une situation de référence qui définit ses repérages déictiques et donc sa valeur référentielle, condition qui, dans ma terminologie, est contenue dans la définition même de l'énoncé.
} 
appartenant au champ de la concession, bien connu pour être un lieu d'expression dialogique par excellence.

\section{Quand même et quand bien même}

Quand même et quand bien même sont deux marqueurs formellement proches, mais de statut morphosyntaxique différent. Ils engagent cependant les mêmes opérations énonciatives de base et illustrent à merveille l'un des postulats de la TOE selon lequel «toute forme synthétique complexe - à savoir une forme que l'on peut appréhender en termes de constituants, dont on peut retracer en diachronie la genèse - conserve, au moins en partie, les propriétés fonctionnelles de ses unités constitutives» 5 .

En effet, sans être à proprement parler compositionnel, le signifié de quand même repose sur deux opérations énonciatives qui sont respectivement portées par chacun de ses deux formants : une opération de parcours sur une classe d'occurrences temporelles exprimée par quand ${ }^{6}$ et une opération de repérage par identification exprimée par $m e ̂ m e e^{7}$. Etymologiquement le parcours opère sur la classe des instants $t$; l'usage a élargi les emplois pour couvrir tout ensemble de procès, temporellement situés, susceptibles de servir de cadre circonstanciel à la relation prédicative en cours. Quant à l'opération d'identification, elle présuppose qu'une valeur de la relation prédicative a préalablement été stabilisée, à laquelle puisse être identifiée la prédication en cours. Le dialogisme d'un énoncé incluant quand même consiste donc à exploiter ces deux opérations de la manière suivante : l'énonciateur primaire reprend et valide un énoncé précédent après avoir envisagé tout un ensemble d'énoncés opposés ou contraires, et il donne explicitement à entendre que son assertion représente une nouvelle prise en charge, plus consciente et plus critique, de l'énoncé initialement posé. Illustrons ce fonctionnement par l'exemple suivant :

5 Lebaux et Mee Rhee 2000 : 70. Pour l'étude de quand même, voir Mellet et Ruggia, sous presse et Veland 1998.

${ }^{6}$ Quand appartient à l'ensemble des termes en $q u$ - issus du thème indo-européen $* \mathrm{k}^{\mathrm{w}}$ - dont la fonction fondamentale est précisément d'exprimer un parcours sur une classe d'occurrences; ces termes se réalisent dans les différentes langues indo-européennes soit comme interrogatifs, soit comme indéfinis selon la modalité retenue pour trouver une issue à ce parcours ; ils fournissent aussi, secondairement, des subordonnants : voir Le Goffic 1994.

${ }^{7}$ Rappelons que dans la TOE l'opération de repérage connaît trois valeurs de base : l'identification, la différenciation et la rupture, auxquelles peuvent s'ajouter des valeurs composites. 
[1] Ça m'étonnerait qu'elle ne rentre pas de toute la nuit. Notez que ça lui arrive quelquefois; elle a une amie chez qui elle va coucher quelquefois. Mais ça m'étonnerait quand même. (Le Clézio, Le Procès)

Le mouvement argumentatif se décompose ainsi :

- Ça m'étonnerait qu'elle (...) : première assertion de $p$

- Notez que ça lui arrive quelquefois; (...) quelquefois: parcours d'événements temporellement situés contrariant $p$ (rôle de l'indéfini)

- Mais ça m'étonnerait quand même : réorientation argumentative (mais) permettant d'asserter à nouveau $p$

Dans ce cas tous les énoncés constituant la séquence argumentative propre à l'emploi de quand même sont explicités. Ils sont attribuables à un seul et même énonciateur, on a donc affaire à de l'auto-dialogisme.

Dans l'exemple [2] la première énonciation de $p$ est sans doute attribuable à l'interviewer (sous forme interrogative : «Est-ce que cette politique fait du mal au PS ?»), mais elle n'est pas retranscrite en tant que telle dans l'article ; on en trouve cependant une trace développée dans le chapeau de l'article (alarme, stress). Le parcours des circonstances contrariant $p$ est explicite. La réorientation argumentative est introduite par un pour autant dont on notera qu'il n'est pas suivi de la négation ${ }^{8}$. Enfin le journaliste verbalise la prise en charge assertive de $p$ par l'énonciateur (admet) :

[2] La politique de «l'ouverture », vue du Parti socialiste, c'est une sorte d'alarme qui se déclenche à intervalles réguliers et provoque un certain stress, même si personne ne s'en inquiète plus vraiment.

(...) Pour Manuel Valls, (...), la ficelle de l'ouverture est devenue très grosse. «Comme toujours, il s'agit d'accréditer l'idée selon laquelle Nicolas Sarkozy est un homme ouvert, alors qu'à l'opposé le PS est devenu sectaire. Mais l'opération tourne à vide. La preuve : ce sont toujours les mêmes noms qui circulent », souligne le député de l'Essonne. Pour autant, $\mathrm{M}$. Valls admet que les alertes récurrentes à l'ouverture « font quand même du mal au PS ». (Le Monde, 23 décembre 2008)

Le plus souvent une ou deux étapes du processus argumentatif restent elliptiques et seule la présence de quand même oblige à reconstruire une première énonciation de $p$, que le contexte permet d'attribuer à tel énonciateur particulier ou à un ensemble d'énonciateurs plus ou moins précis. Ainsi ce titre d'un article du Monde en date du 18 décembre 2008 :

[3] Champagne quand même !

\footnotetext{
${ }^{8}$ Cf. l'analyse de Geneviève Salvan et Sylvie Mellet dans Mellet (dir) 2008 : 136-160.
} 
Cette exclamation mimant les formules rituelles votives de soirs de fête titre un descriptif des diverses marques de champagne (qualité, prix, etc.). Pas d'énoncé antérieur, donc. Mais l'emploi du connecteur oblige à reconstruire le parcours argumentatif suivant :

- [Pensons au] champagne [pour les fêtes] : énoncé de la proposition $p$, contextuellement pertinent vu la date de l'article ;

- Nous sommes en période de crise et de diminution du pouvoir d'achat : contre-argument, lui aussi contextuellement saillant, contrariant $p$;

- [Pensons au] champagne quand même : élimination de l'altérité ainsi ouverte par le parcours des circonstances contraires à $p$ et maintien de la proposition $p$ explicitement identifiée, grâce au seul connecteur, comme reprise d'un énoncé antérieur.

Qu'en est-il de quand bien même ? Selon le principe rappelé au début de cette section, son signifié doit cumuler ceux de ses trois formants, c'est-à-dire ajouter à la valeur de quand et de même celle de l'adverbe bien. A dire vrai, si l'on s'en tient à l'analyse proposée par Culioli ${ }^{9}$, celle-ci est largement redondante avec les deux autres. En effet, bien suppose une altérité préconstruite (c'est-à-dire la reconnaissance d'au moins deux valeurs possibles concurrentes) et identifie la valeur de la relation prédicative qu'il soutient à l'une des deux valeurs préconstruites, généralement sur le ton de la confirmation. Il s'agit donc d'une particule assertive qui pose $p$ sur fond de coexistence $\left(p, p^{\prime}\right)^{10}: p$ a déjà été envisagée, voire énoncée, puis elle a été soumise à questionnement avant d'être définitivement assertée ${ }^{11}$. L'exemple [4] en donne une illustration claire, dans lequel l'altérité est explicitement préconstruite par le contexte antérieur :

[4] La ministre de la santé met en avant l'augmentation de 3,2\% de l'enveloppe budgétaire de l'hôpital (...), mais aussi les 7500 places nouvelles et les 5200 embauches réalisées de 2003 à 2007. Pourtant en novembre, dans une démarche inédite, l'ensemble des présidents des comités consultatifs médicaux des 37 hôpitaux de l'AP-HP ont dénoncé «l'étranglement financier délibéré » de leurs établissements et des «restrictions budgétaires sans objectifs médicaux », aboutissant à « une paupérisation de nos hôpitaux et à un découragement de l'ensemble des personnels ». Qui a raison ? «Les deux affirmations sont exactes, répond Edouard Couty, ancien directeur des hôpitaux au ministère, (...). Il y a bien une augmentation au niveau macroéconomique, mais depuis 2008, la tarification à l'activité est devenue la seule source de financement pour les

\footnotetext{
${ }^{9}$ Culioli $1990: 157-168$.

${ }^{10}$ On rappelle que, dans la TOE, $\mathrm{p}$ ' symbolise le complémentaire de $p$, i.e. non- $p$ et autres-que-p.

${ }^{11}$ Cf Morel 1996 : 42.
} 
activités de court et moyen séjours, ce qui a plongé tous les CHU dans le rouge. » (Le Monde, 31 décembre 2008)

Le connecteur adverbial quand bien même est en outre un connecteur intégratif qui «cheville ${ }^{12}$ deux propositions autour d'une circonstance construite par élimination ou absorption de l'altérité au terme d'un parcours sur l'ensemble des circonstances envisageables. Soit l'exemple [5] :

[5] Les lois dites de bioéthique (1994, 2004), opposent des garde-fous aux prouesses scientifiques. Avec le respect de la dignité humaine. L'anonymat du don. La gratuité. La commercialisation du vivant est ainsi interdite. Ovules et spermatozoïdes ne se négocient pas, quand bien même seriez-vous prix Nobel ou miss Monde. (Ouest France, 5 janvier 09)

- L'énonciateur primaire $\oint_{0}$ asserte une proposition à portée générale (Ovules et spermatozoüdes ne se négocient pas)

- Il assortit cette assertion d'un cadre circonstanciel qui la renforce en prenant en compte l'objection d'un autre énonciateur qui, au terme d'un parcours de toutes les circonstances envisageables (quand) retiendrait l'hypothèse (conditionnel) la plus défavorable à la validation de la principale ; cette hypothèse est clairement attribuée à un autre énonciateur grâce à l'inversion du sujet (seriez-vous) qui manifeste le désengagement partiel de l'énonciateur primaire et grâce au pseudo-dialogue instauré par le vouvoiement (et déjà sous-jacent dans la forme négative de la principale); notons aussi que tout en se faisant l'écho d'une pensée eugéniste diffuse, elle prend ici la forme d'un énoncé clairement articulé et actualisable.

- $\mathscr{S}_{0}$ reprend donc cette hypothèse pour confirmer (bien) que son assertion reste valide: même permet de résorber l'altérité en intégrant à la classe des circonstances favorables à $p$ un cas de figure extrême qui paraissait devoir créer une exception.

A travers l'ensemble des opérations énonciatives ainsi mises en place on voit se construire un dialogisme responsif, de nature ambiguë d'ailleurs puisqu'il peut aussi bien être interdiscursif qu'interlocutif anticipatif (le contexte journalistique faisant sans doute pencher la balance en faveur de la première interprétation). Récapitulons : les opérations énonciatives dont les marqueurs sont la trace sont - le parcours sur une classe d'occurrences,

- la prise en compte de l'altérité, - puis la résorption de celle-ci par identification de l'occurrence autre (relevant $a$ priori de $p^{\prime}$ ) à l'ensemble des occurrences favorables (relevant de $p$ ).

Notons aussi l'importance, pour passer de ces opérations à l'interprétation dialogique, des modalités de la prise en charge énonciative qui permet d'attribuer une source à chacun des énoncés en présence et de positionner l'assertion de $\mathscr{S}_{0}$ par rapport à un discours autre.

${ }^{12}$ Terminologie empruntée à P. Le Goffic. 
Bien sûr, tous les emplois de la locution quand bien même ne s'articulent pas à un contexte aussi explicite : en lieu et place du conditionnel - majoritaire cependant -, la subordonnée peut accueillir à peu près tous les temps de l'indicatif, y compris le futur. L'inversion du sujet n'y est pas systématique : l'ordre /sujet-verbe/ accompagné de l'indicatif manifeste alors l'engagement énonciatif de $\mathscr{S}_{0}$ qui prend en charge l'énoncé secondaire (ou « rapporté ») : on a donc affaire soit à du pur auto-dialogisme, soit à du dialogisme interdiscursif pour lequel $\mathfrak{S}_{0}$ souscrit à l'opinion commune, s'intègre à la communauté des ON-locuteurs ${ }^{13}$. L'ordre des propositions peut admettre l'antéposition de la subordonnée; celle-ci devient alors une concessive logique très proche des concessives en bien que ${ }^{14}$. Comme ces dernières, la subordonnée en quand bien même peut être purement thématique [6] ou être intégrée au rhème et en supporter le focus [7], voire constituer une assertion indépendante [8] :

[6] Quand bien même l'heure est aux économies, la crise, qui est sur toutes les lèvres et dans toutes les bourses, n'a pas eu raison des illuminations. (Presse Océan, 31 décembre 08, accroche-résumé en début d'article)

[7] Or, « son rôle [de la Commission Européenne] consiste à faire des propositions quand bien même celles-ci déplaisent aux capitales », insiste l'un de ses membres, qui regrette un manque d' «ambition» de l'institution. (Le Figaro, 30 décembre 08 ; noter l'absence de virgule avant la subordonnée)

[8] Pas question, annonce la municipalité, d'engager des fonds publics à la légère alors que L'Avventura demande 176000 euros de subvention annuelle. Quand bien même cela représenterait $25 \%$ de moins que les 233 000 euros alloués jusque-là au Jean-Vigo. (Sud Ouest 22 décembre 08)

Mais quoi qu'il en soit de ces variations contextuelles, dans tous les cas que nous avons relevés ${ }^{15}$, le dialogisme fonctionne par reprise d'un énoncé susceptible de contrarier l'assertion de l'énonciateur primaire et par réintégration de cet énoncé au sein de la classe des circonstances compatibles avec le propos de $\mathscr{S}_{0}$ : soit une altérité reconnue et assimilée.

${ }^{13}$ Terme emprunté à Anscombre (voir par ex. 2005), mais inspiré aussi du concept de ON-vérité de Berrendonner..

${ }^{14}$ Exemple : «Quand bien même j'ai des douleurs et je manque d'entraînement, je vais tout faire pour tirer mon épingle du jeu. » (L'Equipe, 20 décembre 08).

${ }^{15}$ Relevé de la presse française parue entre le 5 décembre 2008 et 5 janvier 2009, grâce à la base Factiva. 


\section{La comparaison avec altérité}

Les formes de la comparaison sont multiples ; celle qui m'intéresse ici est la comparaison avec altérité déjà signalée par J. Bres comme porteuse de dialogisme (Bres 2007 : 44-45) ${ }^{16}$. Elle s'exprime dans une structure corrélative, complète ou incomplète en surface, mais qui, dans tous les cas, confronte la proposition $p$ à l'une des propositions autres qui font partie de son complémentaire notionnel, $p$ '.

Comme on l'a déjà dit en effet, dans le cadre de la TOE tout domaine notionnel génère un ensemble de propositions $p$ exprimant la propriété définitoire de la notion (et, à ce titre, identifiables les unes aux autres) et un ensemble complémentaire de propositions $p$ ' contraires à $p$ ou qui, tout en ayant un rapport avec $p$, sont jugées différentes de $p$, non identifiables à $p^{17}$. Dans ce cadre, la comparaison avec altérité consiste donc pour l'énonciateur à se placer d'abord dans une position décrochée qui lui permet d'envisager l'ensemble du domaine notionnel structurant la propriété qui fera l'objet de la relation prédicative, à parcourir les classes d'occurrences qui instancient respectivement $p$ et $p$ ', puis, au terme de ce parcours, à retenir et asserter $p_{i}$ de préférence à une autre proposition concurrente.

[9] L'attachement aux églises de village relève davantage de la mémoire que de la pratique. L'épiscopat réfléchit à la manière de les faire vivre. (L'Est Républicain, 9 novembre 2008)

[9'] L'attachement aux églises de village relève davantage de la mémoire que de tout autre chose.

Le parcours sur la classe d'occurrences est explicité en [9'] par l'indéfini tout ; l'altérité l'est aussi. En [9], l'altérité est préconstruite et la valeur p' (i.e. la pratique) est déjà stabilisée. Une telle préconstruction suppose donc la présence dans un contexte antérieur, entendu au sens large, d'un énoncé - ou, du moins, d'un énonçable - qui a préalablement proposé la sélection de la valeur $p$ ': c'est à un tel énoncé que répond nécessairement [9], l'altérité étant source d'un dialogisme dont le contexte ne permet pas ici de déterminer s'il est intralocutif (auto-dialogisme), interlocutif ou interdiscursif.

En [9'] le dialogisme est moins net parce que la valeur de la proposition $p$ ' n'est pas stabilisée, non plus que la relation intersubjective qu'elle présuppose. Ce n'est donc pas un énoncé qui est mis en écho dialogique, mais une classe ouverte d'énoncés virtuels. Néanmoins ceux-ci peuvent toujours être reconstruits et même si leur inventaire n'est pas exhaustif, la formulation représente l'ensemble des énoncés susceptibles d'avoir été mis en avant par

${ }^{16}$ Voir aussi les analyses de pour autant et de néanmoins dans Mellet (dir.) 2008.

${ }^{17}$ D'où les notations symboliques $\left(p, p^{\prime}\right)$ pour le domaine notionnel et (non- $p$, autre-que-p) pour le complémentaire 
l'énonciateur lui-même ou par d'autres pour expliquer l'attachement avéré aux églises de village.

L'effet dialogique des comparaisons est souvent renforcé par d'autres procédés grammaticaux, notamment le détachement avec l'aide d'un présentatif et/ou la négation polémique :

[10] Si la pensée de Marx est révolutionnaire, c'est moins parce qu'elle est violente que copernicienne : elle est un geste de dévoilement théorique radical, une déconstruction des systèmes civilisationnels. (L'Humanité 5 janvier 2008)

[11] «Notre système territorial marche sur la tête », a de nouveau affirmé le chef de l'Etat, pour qui «la règle générale c'est l'enchevêtrement» et que «tout le monde se mêle de tout et personne n'est responsable de rien». "Le plus grave», selon Nicolas Sarkozy, «n'est pas tant cette confusion des compétences que l'irresponsabilité à laquelle elle conduit, et c'est particulièrement vrai en matière de fiscalité locale ». (AFP 7 janvier 2008)

Il faut ici insister à nouveau sur le rôle des prises en charge énonciatives explicites ou implicites qui font passer de l'altérité au dialogisme. L'altérité est en effet constitutive de la construction même d'un domaine notionnel : partant d'une notion qui est une représentation complexe de propriétés physicoculturelles et qui n'est définissable qu'en intension, de manière quasi tautologique, la construction du domaine notionnel se fait grâce à la fragmentation de cette représentation continue et à son instanciation dans des occurrences qui, seules, permettent d'appréhender réellement la notion à travers des opérations d'identification et de différenciation: d'un fond qualitatif indifférencié, on passe ainsi à des formes différenciées dont certaines incarnent la notion et constituent l'intérieur du domaine tandis que d'autres ne possèdent pas - ou possèdent de manière altérée - la propriété définitoire de la notion et constituent donc l'extérieur du domaine ou sa frontière. Ce n'est qu'à ce stade de construction de la représentation que quelque chose peut être prédiqué de quelque chose. L'altérité est donc, ici aussi, constitutive ; elle est inhérente à notre représentation du monde et à notre système de référenciation: toute prédication, notamment de type existentiel, nécessite en effet qu'un énonciateur parcourt l'entier du domaine pour y sélectionner une valeur de la relation prédicative. Dans l'assertion positive, ce parcours ne laisse que fort peu de traces : la relation prédicative donne naissance, par ancrage sur une situation de référence, à un énoncé qui est pleinement et unilatéralement pris en charge par le sujet énonciateur : celui-ci s'engage sur la validité de la relation et, ce faisant, tente d'effacer au maximum l'altérité constitutive de toute nomination : l'altérité est masquée.

En revanche, dès que l'énoncé comporte des marqueurs qui exhibent les traces de cette altérité, se pose aussitôt la question de la prise en charge énonciative : si 
$\mathscr{S}_{0}$ n'asserte pas purement et simplement $p$, mais suggère, sollicite en contrepoint $p$ ', sachant qu'il est difficile de prendre en charge simultanément l'une et l'autre propositions, la question du statut énonciatif de $p$ ' se pose immanquablement; et plus les marqueurs ont un lien fort avec l'altérité, plus la question se pose avec insistance et appelle à réception la quête d'un second énonciateur ${ }^{18}$.

Le survol rapide de quelques autres marqueurs bien connus pour leur affinité avec le dialogisme va tenter de confirmer ce fonctionnement.

\section{De quelques autres marqueurs de parcours et d'altérité. 4.1 Les hypothétiques en si}

Il ne saurait être question de proposer ici l'analyse approfondie de toutes les variétés d'hypothétiques en si; la littérature sur le sujet est extrêmement abondante et la contribution de Michèle Monte ici même illustre la complexité du problème dès qu'on veut bien s'attaquer sérieusement à la variété des exemples attestés. Je voudrais donc seulement rappeler quelques éléments justifiant la description qui est donnée de ces subordonnées dans la $\mathrm{TOE}^{19}$.

Il existe à l'évidence en français - mais aussi dans de nombreuses autres langues - une parenté formelle entre l'interrogation et le système hypothétique ${ }^{20}:$ le partage d'un même marqueur si d'enchâssement subphrastique en est un premier indice. Le recours possible à une interrogative pour exprimer la protase du système hypothétique en est un autre. Les marqueurs étant la trace en discours d'opérations mentales dûment répertoriées, cette parenté formelle repose nécessairement sur un fonctionnement énonciatif commun qui ne saurait se réduire à un effet pragmatique. En l'occurrence, il s'agit d'une opération de parcours sur l'ensemble du domaine notionnel, qui, après avoir balayé le champ des possibles, cherche une issue en faisant appel au coénonciateur dans le cas de l'interrogation ou se stabilise provisoirement sur une valeur de la relation prédicative dans le cas de l'hypothétique : là aussi le coénonciateur est sollicité pour accepter cette valeur comme point de départ de l'assertion de $q$, qui constitue l'apodose du système si $p$, $q$.

Encore une fois, qui dit parcours dit nécessairement position décrochée de l'énonciateur lui permettant d'envisager comme équipossibles toutes les occurrences du domaine ${ }^{21}$ et de prendre en compte l'altérité constitutive de

${ }^{18}$ Bien évidemment ce second énonciateur peut être une seconde facette du locuteur.

${ }^{19}$ Voir notamment les travaux de Sarah de Voguë.

${ }^{20}$ Cf. Haiman 1978 et 1986, Le Goffic 1994.

${ }^{21}$ Cet ensemble pouvant, bien sûr, se réduire à deux occurrences et offrir une simple alternative entre deux valeurs préconstruites (par ex. "faire beau » $v s$ «ne pas faire beau »). 
celui-ci. Mais l'hypothèse manifeste un rapport complexe à cette altérité : d'un côté elle signale explicitement que la validation de $p$ est suspendue (même si c'est de manière très momentanée, comme dans le cas des subordonnées en si concessives ou récapitulatives); d'un autre côté elle manifeste un engagement assertif indéniable de l'énonciateur, la preuve en étant qu'on ne peut pas dire «s'il fait beau demain ou s'il ne fait pas beau »/ «s'il faisait ou ne faisait pas beau demain » contrairement à ce que permet le subjonctif («qu'il fasse beau demain ou pas »). On a donc une altérité sous-jacente, provisoirement mise de côté au profit d'un acte assertif conditionné et conditionnant : dans [si $p, q], p$, en position thématique, sélectionne $q$, mais l'assertion rhématique de $q$ oblige à réévaluer $p$ : les caractéristiques modales et sémantiques de $q$ contraignent rétroactivement la valeur de $p$ qui peut s'en trouver précisée [12], expliquée [13] ou au contraire limitée, minimisée [14], voire réfutée [15] :

[12] Si [Corneille] était sublime, il l'était alors dans le sens et selon la mode de son temps. (Sainte Beuve, Port-Royal, cité par Patard et Vermeulen)

[13] Si la pensée de Marx est révolutionnaire, c'est moins parce qu'elle est violente que copernicienne (déjà cité sous [10])

[14] S'il est riche, il n'est pas milliardaire (ex. fabriqué emprunté à S. de Voguë)

[15] Si Rieux avait été plus frais, cette odeur de mort partout répandue eût pu le rendre sentimental. Mais quand on n'a dormi que quatre heures, on n'est pas sentimental. (Camus, La Peste)

Le dialogisme volontiers associé aux hypothétiques trouve donc sa source principale dans cette prise en charge explicitement momentanée de $p$ sur fond de $\left(p, p^{\prime}\right)$ : si l'assertion est provisoire, c'est que le sujet énonciateur a lui-même envisagé d'autres valeurs possibles pour la relation prédicative ou qu'il adopte pour un temps le point de vue d'un autre énonciateur pour l'évaluer à l'aune de l'assertion de $q$. Comme le montre Michèle Monte, le coénonciateur est nécessairement associé à cette prise en charge dans une co-construction de la relation $[$ si $p, q]$.

[16] Le chapeau, noir lui aussi et au large bord plat avait roulé sur la pente à l'envers, ridicule et inoffensif. Si je précise inoffensif, c'est parce que cette sorte de chapeau, depuis quelques années, était toujours portée par des êtres effrayants, au rire éteint, et qui nous méprisaient et nous opprimaient. (Pierre Magnan, Un grison d'Arcadie, p.16)

Dans cet exemple, la subordonnée en si reprend une assertion préalable, pour l'expliquer et en évaluer la portée exacte. La valeur de la proposition $p$ est donc provisoirement suspendue pour répondre à l'éventuelle interrogation d'un énonciateur qui n'en comprendrait pas le bien-fondé et qui susciterait un 
parcours sur les autres propriétés attribuables à un chapeau et, à ses yeux, plus appropriées : le dialogisme, ici interlocutif entre narrateur et narrataire, fait entendre un questionnement qu'on peut gloser ainsi : "pourquoi inoffensif ? Je ne comprends pas la pertinence de ce qualificatif ; pour un chapeau j'aurais pensé à d'autres propriétés. » L'altérité notionnelle est donc relayée par une prise en charge énonciative qui assure le dialogisme de l'énoncé. On retrouve très exactement ici le fonctionnement en "mini séquences conversationnelles mettant en scène une séquence question-réponse ${ }^{22}$ reconnu aux hypothétiques. Dans le cas d'une subordonnée en tête de phrase, la prise en charge énonciative donne lieu à la construction d'un topique plus ou moins imposé au coénonciateur : le contenu de la proposition $p$ est présupposé connu, ou du moins pouvant être intégré à l'univers de connaissance du coénonciateur de manière à permettre l'assertion de $q$ dans un cadre argumentatif adéquat. L'exemple suivant en donne une illustration paradoxale, mais à nos yeux probante :

[17] Et pour Mariastella, si c'était bien son deuxième emploi, puisqu'elle avait été durant tant d'années ménagère (...), ce fut aussi son premier amour. (Andrea Camilleri, L'odeur de la nuit, traduit par Serge Quadruppani).

Rien dans le contexte romanesque antérieur ne permet de savoir que Mariastella a été femme de ménage ; mais la fonction «topique» ou «cadrative » de la subordonnée en si est néanmoins confirmée par l'insistance de la subordonnée en puisque qui, elle aussi, présuppose connu le fait rapporté.

Mais le dialogisme peut trouver à s'alimenter dans d'autres éléments du système hypothétique : on retrouve alors le cumul de marqueurs à orientation dialogique que nous avons déjà noté à propos des comparaisons. En premier lieu signalons le choix de la relation entre $p$ et $q$ qui fait entendre un topos ou, du moins, des inférences communément admises et dont l'orientation argumentative est exploitée [18] ou contrecarrée [19] :

[18] S'il est riche, il se montrera généreux (ex. fabriqué d'après Patard et Vermeulen)

[19] S'il est riche, il est pingre aussi et ne nous donnera rien

Ces deux énoncés font entendre l'énoncé sous-jacent attribuable à un ONlocuteur auquel s'associe $\mathscr{S}_{0}:$ : quand on est riche on peut aisément se montrer généreux ».

L'emploi de certains tiroirs verbaux renforce encore les potentialités dialogiques du système hypothétique. Ainsi, dans le cas d'un irréel, l'altérité sollicite un

${ }^{22}$ Charolles 2003 commentant Haiman et, au-delà, Jespersen. 
préconstruit reconnu comme tel par l'énonciateur et le coénonciateur : si les poules avaient des dents, si j'étais toi, s'il avait fait beau présupposent que les poules n'ont pas de dents, que je ne suis pas toi, qu'il n'a pas fait beau : autant d'énoncés partagés sous-jacents qui alimentent le dialogisme de la structure.

Cette remarque nous conduit à terminer par un bref rappel sur l'imparfait français, à propos duquel notre position diffère quelque peu de celle de Bres et Patard exprimée ici même.

\subsection{L'imparfait, un temps au potentiel dialogique programmé en langue}

Dans une perspective énonciative, la représentation d'un procès à l'imparfait nécessite une double opération de repérage: translation des coordonnées énonciatives (permettant de situer le procès dans le passé ou dans un monde fictif par différenciation avec 'Sit $t_{0}$ ) et, à partir du nouveau repère ainsi construit, appréhension du procès saisi en cours de développement (ou, si l'on préfère, en incidence sur décadence) par identification partielle entre l'intervalle de temps occupé par la situation repère et l'intervalle du procès. La représentation du procès à l'imparfait repose donc sur un double ancrage, la situation repère translatée se superposant à la situation d'énonciation primaire. Ce double repérage est définitoire de la valeur en langue de l'imparfait; on notera qu'il confère à la situation translatée la fonction de repère secondaire instaurant le point de vue sur le procès : sans cette promotion du repère en point de vue, pas de saisie interne, pas de vision sécante, pas d'ouverture à droite du procès, donc pas de fondement à toutes les valeurs aspectuelles bien connues de l'imparfait. Ce point de vue est celui d'un sujet énonciateur à même de valider la relation prédicative à l'imparfait; il introduit l'altérité énonciative au cœur même de ce tiroir verbal ${ }^{23}$.

Pour Bres et Patard, le point de vue secondaire n'existe de manière stable qu'au conditionnel, en lien avec la visée prospective de celui-ci. C'est à mon avis une erreur qui consiste à confondre la permanence d'un effet discursif avec son inscription dans le signifié en langue de la forme. Car s'il est vrai que la visée prospective oblige à toujours mobiliser le repère secondaire pour construire la représentation du procès au conditionnel, il n'en est pas moins vrai que la construction du point de vue interne au procès est définitoire de l'imparfait et, à ce titre, susceptible d'apparaître dans tous les types d'emplois de ce tiroir verbal. Le dédoublement énonciatif est obvie dans tous les emplois modalisés instaurant un repère fictif, comme dans les exemples d'irréel mentionnés ci-dessus mais aussi dans les emplois dits ludiques et autres emplois modaux ; il est tout aussi évident dans les emplois en discours rapporté [20], et notamment à la frontière entre la narration et le discours indirect libre $[21]^{24}$ :

[20] - Il n'est pas malade. Il est mort !

\footnotetext{
${ }^{23}$ Voir Mellet 2000.

${ }^{24}$ Ibid.
} 


\section{- Comment tu sais?}

L'Espagnol leva les yeux au ciel : comment il savait ! Ses dix-sept ans fleuris : Santander, Teruel, Irun le siège de Barcelone... Comment il savait! Quelle question! (Pierre Magnan, Les Atrides, p. 12)

[21] Il n'en pensait pas un mot. Il aurait préféré les constatations faites, le cadavre emporté, le coupable inopinément arrêté, alors que, derrière un arbre, il espionnait les investigation de la police. C'était un rêve. Au travail! (Id. pp. 15-16)

Mais les emplois les plus banals de l'imparfait descriptif ne sont pas exempts non plus de cette altérité énonciative ; dans l'exemple suivant c'est le même repère translaté qui sert de point de vue pour construire et les deux conditionnel et les quatre imparfaits, et son statut est le même quel que soit le tiroir verbal utilisé :

[22] Quand elle se tourna pour refermer la serrure à clé, la clarté de sa torche la découpa en silhouette sur l'obscurité. La vieillarde qu'elle serait un jour était déjà imprimée en elle et la courbait vers la terre. Pour un peu je me serais avancé pour la soutenir sur cet escalier verglacé où elle risquait de trébucher, tant elle me paraissait fragile, cassable. (Pierre Magnan, Un grison d'Arcadie, p. 160)

Et comment construire un imparfait narratif si le narrateur omniscient conserve le monopole d'un point de vue unique et surplombant sur le procès décrit ?

Donc, à partir du moment où il n'existe aucun emploi de l'imparfait qui refuse le surgissement du point de vue énonciatif second, on peut conclure que l'imparfait fait bien partie, au même titre que le conditionnel, de ces morphèmes pour lesquels l'expression de l'altérité énonciative est définitoire du signifié en langue. Simplement, son actualisation en dialogisme discursif ne s'exprime pas toujours fortement. Les différentes interprétations contextuelles aboutissent tantôt à la saillance, tantôt à l'atténuation de l'effet dialogique. L'imparfait n'est pas le seul marqueur dans ce cas; il en va exactement de même pour la négation : celle-ci, que nous n'avons pas la place de traiter ici, est évidemment un marqueur d'altéritée ${ }^{25}$; il n'empêche qu'à côté des négations polémiques certaines négations descriptives ne laissent quasiment pas entendre l'énoncé positif sous-jacent ${ }^{26}$. Une subordonnée en si n'a pas exactement la même

${ }^{25}$ Toutes les analyses l'ont relevé, quel que soit le cadre théorique adopté. Pour le dire trivialement, on considère que tout énoncé négatif prend appui sur - et fait entendre - l'énoncé positif correspondant. Pour une analyse dans le cadre de la TOE, voir Culioli 1988.

${ }^{26}$ Voir par exemple Nølke (1993). A noter qu'entre les deux il y a des négations descriptives dialogiques; ainsi de cet énoncé relevé dans le deuxième paragraphe du roman Les Atrides : «Aucun chien n'aboyait». Le narrateur met 
évidence dialogique selon qu'elle est purement hypothétique ou qu'elle est concessive ou adversative. Il en va de même pour le conditionnel lui-même, selon qu'il se trouve dans l'apodose d'un système hypothétique ou qu'il s'agisse d'un conditionnel «médiatif » ou «journalistique ». On citera aussi le modal pouvoir dont le signifié propre consiste à ouvrir une alternative et à conférer un poids égal (une équiprobabilité) à chacune des branches de celle-ci, à chacun des chemins qui s'ouvrent à l'énonciateur: entièrement ancré sur l'altéritée ${ }^{27}$, le verbe pouvoir a généralement un fort potentiel dialogique, comme l'attestent ses combinaisons fréquentes avec d'autres marqueurs tels que bien, quand même, si, le conditionnel, etc. :

[23] L'hyperpuissance de l'armée israélienne peut bien aujourd'hui écraser les miliciens islamistes, il est peu probable que l'Autorité palestinienne en tire mécaniquement le bénéfice. Le Hamas ne disparaîtra pas comme par magie sous les tirs d'obus (Le Monde, 6 janvier 2009)

[24] Même si, à la vue des prochaines données macroéconomiques qui annoncent pour 2009 une récession plus importante que prévu, la chancelière allemande pourrait bien revoir ses positions, [...], cela portera sur l'ampleur de l'engagement des pouvoirs publics allemands et non sur une quelconque dimension européenne. (Le Monde, 18 décembre 2008)

[25] On pouvait la souiller, la jeter à terre, la tuer : elle gagnerait quand même. (Le Clézio, Déluge)

Et pourtant, il est des emplois dans lesquels ce potentiel dialogique semble s'effacer complètement: ce sont en particulier ceux dans lesquels pouvoir conserve son plein sens lexical de capacité (Tu peux y arriver tout seul).

en place un cadre caractérisé par la plus totale banalité et le vide, l'inexistence de tout élément marquant. Cet énoncé est un clin d'œil aux nombreuses occurrences romanesques du type «Un chien aboyait au loin /dans la nuit » : sur 70 occurrences de la forme aboyait dans les romans du $20^{\text {ème }}$ siècle enregistrés dans la base Frantext, on en compte 10 qui relève de ce patron descriptif ; en voici trois exemples caractéristiques : «Un souffle de vent s'en alla d'arbre en arbre du côté du ruisseau. Quelque nuage aux bords éclatants jetait son ombre en passant sur la lune. Un chien aboyait dans une cour de ferme. » (H. Pourrat, Les Vaillances); "Il eut un oblique regard sur la campagne que la brume du soir estompait. Déjà des lumières s'allumaient au loin. Un chien aboyait et de jeunes garnements se défilaient sournoisement sur le sentier gris de la zone, (Francis Carco : L'équipe : roman des fortifs) ; «Elle resta un moment sans répondre. Les lucioles voltigeaient. Quelque part, un chien aboyait, doux, triste, lointain. (M.E. Coindreau, Lumière d'août).

${ }^{27}$ Cf. Fuchs 1991. 
Ce constat suggère d'ailleurs que la dialogisation d'un marqueur pourrait bien être associée à un processus de grammaticalisation (qu'on pourrait appliquer non seulement au verbe pouvoir, mais aussi à l'adverbe bien ou au connecteur intégratif quand bien même, pour ne citer que ceux que nous avons évoqués dans cet article) : c'est là une hypothèse qui reste à explorer.

\section{Conclusion}

Nous avons examiné, dans une liste qui peut paraître quelque peu hétéroclite, quelques-uns des marqueurs grammaticaux - ou grammaticalisés - qui sont réputés soutenir fortement l'expression du dialogisme.

Nous pensons avoir montré que, derrière leur extrême diversité, se cache un point commun stable : chacun de ces marqueurs sollicite, à sa façon, l'altérité constitutive de toute représentation notionnelle en langue. Chacun d'eux convoque et construit une représentation duale de la notion ou du procès à l'issue d'un parcours sur l'intérieur et sur l'extérieur du domaine ; chacun d'eux laisse ainsi ouverte la possibilité d'un double point de vue - sur la propriété $p$ ou sur son complémentaire $p^{\prime}$ - tout en assurant la prise en charge énonciative de $p$. Cette prise en charge donne, en creux, à $p$ ' le statut d'énoncé sous-jacent, car ce sont les deux facettes de l'altérité qui, à travers les opérations de parcours et de sélection d'une valeur par différenciation des diverses occurrences, puis identification de la «bonne » occurrence, font l'objet de l'acte énonciatif : celuici convoque donc nécessairement un énonciateur secondaire dont le contexte dira s'il est présent, absent, figuré, fictif, double de l'énonciateur primaire, etc.

Si donc notre analyse est juste, il faut conclure qu'il y a bien dans le système linguistique des opérations énonciatives primaires modélisables qui sous-tendent - mieux : qui intègrent à la grammaire de la langue - ce fait dialogique que, dans la lignée de Bakhtine, nous jugeons constitutif du langage humain et qui ne saurait se réduire à une simple mise en scène polyphonique.

\section{Références bibliographiques :}

Achard-Bayle, G. (2006). «Si polysémique et si polyphonique », in L. Perrin (dir.), 407-434.

Anscombre, J.-C. (1990). «Thème, espaces discursifs et représentation événementielle », in J.-C. Anscombre et G. Zaccaria (éds), Fonctionnalisme et pragmatique. A propos de la notion de thème. Testi e studi $76: 42-150$.

Anscombre, J.-C. (2005). «Le ON-locuteur : une entité aux multiples visages », in J. Bres et al. (éds), 75-94.

Bakhine 1952/1979/1984b: «Les genres du discours » in Esthétique de la création verbale, Paris : Gallimard, 265-308. 
Bres, J. (1999). "Vous les entendez ? Analyse du discours et dialogisme », Modèles linguistiques XX, 2 : 71-86.

Bres, J et Rosier, L. (2007). "Réfractions : polyphonie et dialogisme, deux exemples de reconfigurations théoriques dans les sciences du langage francophones », in B. Vauthier (éd.), Bakhtine, Volochinov et Medvedev dans les contextes européen et russe, Slavica Occitania 25 : 437-461.

Bres, J (2007). «Sous la surface textuelle, la profondeur énonciative. Les formes du dialogisme de l'énoncé », in R. Therkelsen, N. Møller Andersen et H. Nølke (éds), Sproglog Polyfoni, Aarhus Universitetsforlag, 37-54.

Bres J. et Nowakowska A. (2006). «Dialogisme : du principe à la matérialité discursive », in L. Perrin (dir.), 21-48.

Bres, J. et al. (éds) (2005). Dialogisme, polyphonie : approches linguistiques, Bruxelles : De Boeck / Duculot.

Culioli, A. (1988). «La négation : marqueurs et opérations », Travaux du Centre de Recherches Sémiologiques 56 : 17-38.

Culioli, A. (1990 et 1999). Pour une linguistique de l'énonciation, t. I et t. III, Paris / Gap : Ophrys.

Fløttum, K. (2005). «Moi et autrui dans le discours scientifique : l'exemple de la négation ne ... pas », in J. Bres et al. (éds), 323-337.

Fuchs, C. (1991). «Polysémie, interprétation et typicalité : l'exemple de pouvoir », in D. Dubois (éd.), Sémantique et cognition: catégories, prototypes, typicalité, Paris : Editions du CNRS, 161-170.

Gaudin, L., Salvan, G. et Mellet, S. (2008). «Pourtant et pour autant», in S. Mellet (dir.), 97-160.

Haillet, P. P. (2002). Le conditionnel en français : une approche polyphonique, Paris / Gap : Ophrys.

Haiman, John (1978). «Conditionals are topics », Language 54 :564-589.

Haiman, John (1986). "Constraints on the form and the meaning of the protasis », in E. Traugott et al. (eds), On Conditionals, Cambridge : Cambridge university Press, 215-227.

Lebaud, Daniel et Mee Rhee, Eun (2000). «Cependant, pourtant et altération d'un dire », in Répétition, Altération, Reformulation, Annales littéraires de l'Université de Besançon 701. Presses Universitaires Franc-Comtoises, 6790.

Le Goffic, Pierre (1994). «Indéfinis, interrogatifs, relatifs : parcours avec ou sans issue », Faits de Langues $4: 31-40$.

Mellet, S. (2000). «A propos de deux marqueurs de bivocalité », in S. Mellet et M. Vuillaume (éds), Cahiers Chronos, 5, «Le style indirect libre et ses contextes », Amsterdam - Atlanta : Rodopi, 91-106. 
Mellet, S. (dir.) (2008). Concession et dialogisme. Les connecteurs concessifs à l'épreuve des corpus. Bern : Peter Lang.

Mellet, S. et Monte, M. (2008). «Néanmoins et toutefois », in S. Mellet (dir.), 55-85.

Mellet, S. et Ruggia, S. (sous presse). «Quand même, à la croisée des approches énonciatives ", in Actes du XXVème Congrès international de linguistique et philologie romanes (Innsbruck, 3-8 septembre 2007).

Merle, Jean-Marie (1999). "Genèse et interprétation des repères hypothétiques en milieu toncal », LINX $41: 61-74$.

Morel, M.-A. (1996). La concession en français, Paris/Gap : Ophrys (« L'essentiel français »).

Nølke, H. (1993). Le regard du locuteur, Paris : Kimé (en part. «Ne ... pas : négation descriptive ou polémique? Contraintes formelles sur son interprétation », pp. 233-258).

Nølke, H. (2001). Le regard du locuteur 2. Pour une linguistique des traces énonciatives, Paris : Kimé.

Patard A. et Vermeulen C. (???). «Essai de représentation de la phrase hypothétique de forme [si $P(I M P), Q(C O N D)]$ », Cahiers Chronos ???

Perrin, L. (dir.) (2006). Le sens et ses voix. Dilaogisme et polyphonie en langue et en discours, Metz : Université Paul Verlaine, Recherches linguistiques ${ }^{\circ}$ 28.

Trévise, Anne (1999). «A propos des repérages fictifs : variété des formes et construction du sens », LINX $41: 39-59$.

Valentin, P. (éd.) (1989). La comparaison. Université Paris-Sorbonne : Linguistica Palatina, Colloqia III.

Veland, Reidar (1998). "Quand même et tout de même: concessivité, synonymie, évolution », Revue Romane 33, 2 : 217-247.

De Voguë, S. (1992). «Si, la syntaxe et le point de vue des opérations », in s.n. La théorie d'Antoine Culioli. Ouvertures et incidences, Paris / Gap : Ophrys, 123-144.

De Voguë, S. (1999). «Le champ des subordonnées dites conditionnelles en français », LINX $41: 93-118$.

De Voguë, S. (2004). «Si, au centre et aux marges de la condition », in C. Hare (coord.), L'hypothèse au miroir des langues. Paris : L'Harmattan, pp. 85117. 